\title{
Research highlights from the 2018 European Respiratory Society International Congress: airway disease
}

\author{
Florence Schleich ${ }^{1}$, Andras Bikov ${ }^{2,3}$, Alexander G. Mathioudakis ${ }^{2,3}$, \\ Melissa McDonnell ${ }^{4}$, Cecilia Andersson ${ }^{5}$, Matteo Bonini ${ }^{6,7}$, Lena Uller $^{8}$, \\ Marco Idzko ${ }^{9}$, Dave Singh ${ }^{10}$, Jose Luis Lopez-Campos ${ }^{11,12}$, \\ Apostolos Bossios ${ }^{13,14}$, Ian M. Adcock ${ }^{7}$, Omar Usmani ${ }^{7}$, \\ Antonio Spanevello ${ }^{15}$ and Sara J. Bonvini ${ }^{7}$
}

Affiliations: 'Dept of Pulmonary Medicine, CHU Sart-Tilman and I3 GIGA Research Group, University of Liège, Liège, Belgium. ${ }^{2}$ Division of Infection, Immunity and Respiratory Medicine, School of Biological Sciences, University of Manchester, Manchester, UK. ${ }^{3}$ North West Lung Centre, Wythenshawe Hospital, Manchester University NHS Foundation Trust, Manchester, UK. ${ }^{4}$ Dept of Respiratory Medicine, Galway University Hospitals, Galway, Ireland. ${ }^{5}$ Unit of Respiratory Cell Biology, Dept of Experimental Medical Science, Lund University, Lund, Sweden. 'UOC Pneumologia, Istituto di Medicina Interna, F. Policlinico Gemelli IRCCS, Università Cattolica del Sacro Cuore, Rome, Italy. ${ }^{7}$ National Heart and Lung Institute, Imperial College London, London, UK. ${ }^{8}$ Unit of Respiratory Immunopharmacology, Dept of Experimental Medical Science, Lund University, Lund, Sweden. ${ }^{9}$ Dept of Pneumology, Medical University of Vienna, Vienna, Austria. ${ }^{10}$ Medicines Evaluation Unit, University of Manchester, Manchester University NHS Foundation Trust, Manchester, UK. ${ }^{11}$ Unidad MédicoQuirúrgica de Enfermedades Respiratorias, Instituto de Biomedicina de Sevilla (IBiS), Hospital Universitario Virgen del Rocío/Universidad de Sevilla, Seville, Spain. ${ }^{12}$ Centro de Investigación Biomédica en Red de Enfermedades Respiratorias (CIBERES), Instituto de Salud Carlos III, Madrid, Spain. ${ }^{13}$ Dept of Respiratory Medicine and Allergy, Karolinska University Hospital, Huddinge, Sweden. ${ }^{14}$ Dept of Medicine, Karolinska Institutet, Stockholm, Sweden. ${ }^{15}$ Istituti Clinici Scientifici Maugeri, Università degli Studi Insubria, Tradate, Italy.

Correspondence: Sara J. Bonvini, Respiratory Pharmacology, National Heart and Lung Institute, Imperial College London, SW7 2AZ, UK. E-mail: sara.bonvini10aimperial.ac.uk

ABSTRACT The annual European Respiratory Society (ERS) International Congress (held in Paris in 2018) was once again a platform for discussion of the highest-quality scientific research, cutting-edge techniques and innovative new therapies within the respiratory field. This article discusses only some of the high-quality research studies presented at this year's Congress, with a particular focus on airway diseases including asthma, chronic obstructive pulmonary disease (COPD), bronchiectasis and cough, as presented through Assembly 5 of the ERS (Airway Diseases: Asthma and COPD). The authors establish the key take-home messages of these studies, compare their findings and place them in the context of current understanding.

@ERSpublications

A review of the high-quality research studies presented at the 2018 \#ERSCongress, with a focus on airway diseases, including asthma, COPD, bronchiectasis and cough, presented through Assembly 5 of @ERStalk http://ow.ly/tQCl30nrPMI

Cite this article as: Schleich F, Bikov A, Mathioudakis AG, et al. Research highlights from the 2018 European Respiratory Society International Congress: airway disease. ERJ Open Res 2019; 5: 00225-2018 [https://doi.org/10.1183/23120541.00225-2018]. 


\section{Asthma}

Basic and translational data

Asthma is one of the most common inflammatory diseases globally and affects over 200 million people worldwide [1]; however, little is known regarding the detailed mechanisms driving the disease [2]. Asthmatic disease shows a high degree of heterogeneity [3] and this topic was elegantly addressed by Prof. Sally Wenzel in her talk "Molecular phenotyping of asthma comes of age" in the symposium "State of the art session: airways disease". In line with this, NAwijn et al. [4] showed, using single cell RNA sequencing, that direct comparison of airway wall cells from asthmatic patients and healthy controls revealed selective loss of well-differentiated ciliated cells accompanied by an increase in basal cells in asthma. Specific inflammatory cell patterns were also observed in asthma and TOKUNAGA et al. [5] presented that CD163, a scavenger receptor expressed on macrophages, was significantly increased in asthma death patients compared to control subjects. They also confirmed in a mouse model of allergic inflammation that airway hyper-responsiveness (AHR) and the number of eosinophils in bronchoalveolar lavage fluid (BALF) were significantly decreased in CD163 deficient mice when compared with control wild-type mice. This indicates an important role for altered phenotypes of macrophages not only in chronic obstructive pulmonary disease (COPD), as previously reported, but also in asthma. It is evident that overlap between the inflammatory response in asthma and COPD exists, especially when it comes to patients who smoke. SiLBERBRANDT et al. [6] reported that, in a severe asthma cohort with predominantly late-onset asthma, airway eosinophilia was significantly associated with a smoking history of $\geqslant 10$ pack-years. They concluded that further research was needed to explore underlying mechanisms driving eosinophilic airway inflammation in severe asthma. Another factor in phenotyping asthma is age of onset and MistRY et al. [7] showed that there are phenotypic differences between early-onset and adult-onset difficult asthma. They showed that early-onset difficult asthma is more common in females and associated with more atopy, functional comorbidity, biologic therapy and healthcare need. Adult-onset difficult asthma meanwhile is more common in males associated with smoking and comorbid lung disease. Mapping clinical phenotypes and correlating them to molecular endotypes and inflammatory patterns will be of great importance to increase our knowledge of mechanisms driving asthmatic disease as well as treatment strategies.

Much focus during the 2018 European Respiratory Society (ERS) International Congress was on the mechanistic understanding of cell-microbe communications in the lung micro-environment and how they promote airway disease or protect patients from it. The symposium "Cross-talk in the lung microenvironment: implications for chronic lung disease" (session 218) focused on different aspects of the cellular communication mechanisms in the lung that participate in health and disease, with presentations by Profs. D. Davies, P. Hansbro, S. Krauss-Etschmann and S. Gabrielsson. The 2018 ERS International Congress also had a "Meet the Expert" session on the topic "Lung microbiome: study design and standardisation" (ME2). Lately, a shift in focus away from microbial involvement in infections and toward a role in physiology has been observed. For a long time the lungs of healthy individuals were considered to be free from microbes and hence to lack a microbiome. However, while the lung microbiota has a low density, the maintenance of small numbers of bacteria seems to be a critical determinant of good health [8]. РотAсZек et al. [9] demonstrated that interleukin (IL)-6, a central cytokine of innate immunity responses, seems to be important for the ability to modulate the adaptive immune system in a non-allergic Type-2 T-helper cell (Th2) direction. They also showed that IL-6 plays a specific role in mediating the development of protective, anti-allergic mechanisms related to prior Acinetobacter lwoffii exposure. Probiotics can be considered nonspecific adjuvants of innate immune response by modulating the Type- 1 T-helper cell (Th1)/Th2 balance. Aimbire et al. [10] showed that probiotic Lactobacillus bulgaricus mediated an anti-inflammatory effect in an experimental mouse model of allergic inflammation. They showed that dendritic cell expression of CD86 as well as the Toll-like 4 receptor was increased after oral administration of $L$. bulgaricus. In addition, L. bulgaricus attenuated eosinophil infiltration, mucus production and specific IgE concentration in serum, and also inhibited secretion of IL-4, IL-5, IL-13 and eotaxin in BALF. In a clinical study DE BOER et al. [11] presented interesting data from the BREATHE study where bacterial lysates showed positive effects in the prevention of asthma exacerbations in patients with severe asthma.

\section{Clinical studies}

Asthma is a heterogeneous inflammatory airway disease and the majority of asthmatics (59\%) treated in general practice are uncontrolled [12]. When physicians recognise poor asthma control, an appropriate change to therapy must be considered. At this year's Congress, VAN DER MeER et al. [13] presented their research on dynamic hyperinflation, which, independent of asthma severity, is associated with poorer overall health, less well-being and impaired activity in daily life. Due to its major impact on activity in everyday life, dynamic hyperinflation should be an important target for treatment in asthma patients in the future. 
It is also well known that asthma is associated with accelerated rate of lung function decline. The relationship between decline and airway inflammation among asthmatics has important therapeutic implications. In a large cohort, BACKMAN et al. [14] found that adult asthmatics with higher levels of blood eosinophils had a history of excess forced expiratory volume in $1 \mathrm{~s}$ (FEV1) decline compared to non-eosinophilic asthmatics, independent of other factors such as inhaled corticosteroid (ICS) use. GRAFF et al. [15] presented similar results and their observations go further, showing that an evolution towards an increase in blood eosinophils over time predicts accelerated FEV1 decline. They further confirmed that this was independent of inflammatory phenotype or ICS treatment category.

A significant proportion of patients with difficult-to-control asthma remained nonadherent to corticosteroid therapy [16]. Alahmadi et al. [17] presented data from the U-BIOPRED study with the aim of investigating the adherence of asthmatics according to the Medication Adherence Report Scale (MARS) and urinary tests. One third of asthmatics reported sub-optimal adherence, while $42 \%$ of the severe asthmatics did not have detectable urinary prednisolone or metabolites. In this study, there was very poor agreement between MARS and urinary testing, suggesting that both approaches should be performed as part of a difficult asthma assessment and are important before prescribing expensive novel biological therapies.

Moreover, multiple comorbidities are associated with asthma control, healthcare utilisation and quality of life (QoL) in difficult asthma, and the data presented by AzIM et al. [18] demonstrated a high prevalence of atopy, obesity, rhinitis and gastro-oesophageal reflux disease (GORD) in the Wessex Asthma Cohort. In the area of personalised medicine there is increasing interest in the identification of treatable traits. The analysis of the Australasian Severe Asthma Web-based Database revealed that treatable traits, such as allergic sensitisation, upper-airway disease, airflow limitation, eosinophilic inflammation and frequent exacerbations, are more common in severe asthma. Ten traits predicted exacerbation risk with the strongest being frequent exacerbations, depression, inhaler-device polypharmacy, vocal cord dysfunction and obstructive sleep apnoea (OSA) [19]. Psychological stress exacerbates clinical symptoms in patients with asthma and stress has been previously shown to amplify the immune response to asthma triggers [20]. The study by BACON et al. [21] provides evidence that acute stress negatively influences the physiology of asthmatics. Stress did not affect the caliber of bronchi but was associated with decreased carbon dioxide production, potentially suggesting hypocapnia and hyperventilation.

There are a lot of treatment options in the management of asthma; however, inhaled glucocorticoids remain a cornerstone because of their efficacy, tolerability and rapid onset of action. Unfortunately, there are patients in clinical practice who do not respond to ICS and predictors of such a poor response, such as the absence of eosinophilic inflammation, have been highlighted previously [22]. MARCON et al. [23] presented a study looking at FEV1 decline in asthma patients treated with ICS. Here, ICS use was associated with increased FEV1 decline among subjects without sensitisation but not in sensitised individuals. This suggests that a long-term ICS therapy may be more effective in preventing FEV1 decline in allergic compared to non-allergic asthma [24].

After monitoring and treating comorbidities, 5-10\% of the asthmatic population remains uncontrolled despite high-dose glucocorticosteroids and bronchodilator medications. Here, we focus on data for biologics presented at the 2018 ERS International Congress. Targeting IgE, omalizumab is able to reduce exacerbations and improve asthma control and QoL in severe allergic asthmatics. The objective of the study presented by HANNANIA et al. [25] was to evaluate the reduction of exacerbation in patients treated with omalizumab according to their blood eosinophil counts (BECs). The reduction in exacerbations was $53 \%$ in patients with BECs greater than 300 per $\mathrm{mm}^{3}$ and $42 \%$ in patients with BECs less than 300 per $\mathrm{mm}^{3}$, suggesting that BEC is not a good predictor of response to anti-IgE.

Monoclonal antibodies targeting cytokines or their receptors may induce the production of anti-drug antibodies. ORtega et al. [26] have looked at the immunogenicity profile of mepolizumab during the Phase III clinical development programme in severe asthma (COSMOS and COLUMBA). There was no correlation between anti-drug antibody titres and change in BECs. Adverse events evaluated as potential systemic allergic reactions were uncommon $(2 \%)$ and unrelated to the study drug in antibody-positive patients. WeNZEL et al. [27] conducted a post hoc analysis of pooled data from the SIROCCO and CALIMA studies on the effect of benralizumab in patients classified according to age of onset $(<18, \geqslant 18$ to $<40$ and $\geqslant 40$ years). They found a higher effect of benralizumab in adult-onset and late-onset asthma in terms of FEV1 improvement or reduction in exacerbations as compared to early-onset asthma. This suggests that adult-onset asthma with less atopy is the prime target population for anti-IL-5 therapy.

Approved for the treatment of moderate-to-severe atopic dermatitis, dupilumab is a fully human, anti-IL-4R monoclonal antibody (mAb) that inhibits IL-4/IL-13. During the Congress, Corren et al. [28] presented their findings that dupilumab was more effective in asthmatics with chronic rhinosinusitis and nasal polyps. In this sub-population, dupilumab significantly improved asthma control and QoL. 
Bronchial thermoplasty (BT) offers an alternative therapeutic option for severe asthmatics with persistent symptoms requiring step five treatment in specialised centres. There is still a lack of data on predictors of response to BT. During this Congress, Torrego et al. [29] reported the results of the real-world BT Global Registry study (including 159 patients). They suggested that healthcare utilisation decreased, clinical outcomes improved and medication usage was reduced at 1 year after BT. FrIX et al. [30] also presented that thermoplasty was well tolerated, with an improvement in pre- and post-FEV1 together with a reduction in exacerbation rate and in the average dose of oral corticosteroids (OCS) in severe non-T2-asthmatics. In addition, GoORSENBERG et al. [31] presented data on BT-treated patients and showed that lung function parameters were unchanged after BT. However, a higher airway resistance was observed in non-responders suggesting that a high resistance at baseline could be a negative predictor for BT response. Furthermore, SANO et al. [32] presented BT data showing that responders to BT exhibited higher R5 and R5-R20 (where R5 is the total respiratory system resistance, measured at $5 \mathrm{~Hz}$ and R20 is the central resistance, measured at $20 \mathrm{~Hz}$ ) at baseline compared to the non-responders. The responders also exhibited a decrease in resistance compared to the non-responders 1 year after BT treatment.

\section{COPD}

\section{Biomarkers}

There is an unmet clinical need in COPD for biomarkers which reflect disease activity (i.e. future exacerbations and rate of lung function decline) or predict treatment responsiveness. Blood eosinophils are surrogate markers for airway eosinophilia [33] and previous studies have showed that patients with higher BECs benefit from a reduction in exacerbations when treated with ICS-containing regimes (ICS/ long-acting $\beta$-agonist (LABA) or ICS/LABA/long-acting muscarinic antagonist (LAMA)). There are still some uncertainties which have to be clarified regarding BEC, as follows: 1) studies are primarily based on Western populations and we do not know if proposed BEC cut-off values (i.e. $>300 \mathrm{cells} \cdot \mathrm{mm}^{-3}$ ) can be generalised for all populations; 2) the stability of eosinophil-low and eosinophil-high phenotypes has to be established; 3) the impact of undiagnosed fungal and parasitic infections needs to be determined; and 4) the effect of previous ICS treatment on BEC and its prognostic value need to be determined. PASCOE et al. [34] presented data from the IMPACT trial which compared ICS/LAMA/LABA to ICS/LABA and LABA/ LAMA treatments. The authors concluded that increasing BECs were associated with increased ICS effects on exacerbation on a continuous scale. Mathioudakis et al. [35] investigated the data from the ISOLDE trial and confirmed that BEC is predictive for ICS response in ICS-naïve COPD patients [36]. Furthermore, direct ICS withdrawal from triple therapy led to increased risk of moderate/severe exacerbations in patients with consistently high BECs in the SUNSET trial [37].

Markers predicting future mortality in COPD are warranted to identify patients at high risk who may benefit from early interventions and close follow-up. WASCHKI et al. [38] presented results from the COSYCONET cohort where serum high-sensitivity troponin I was evaluated in stable COPD patients and it was found that serum levels $>6 \mathrm{ng} \cdot \mathrm{L}^{-1}$ were associated with all-cause mortality even after adjustment for cardiovascular (CV) factors, body mass index (BMI), lung function, modified Medical Research Council (mMRC) dyspnoea score and 6-min walk distance (6MWD) [39]. In addition, Agustí et al. [40] presented data from the ECLIPSE cohort evaluating the Global Initiative for Chronic Obstructive Lung Disease (GOLD) 2017 disease groups (A, B, C and D) and their relation to mortality. The authors reported an association between mortality and the highly symptomatic groups (B and D). This highlights the clinical relevance of symptom assessment as an important therapeutic goal in COPD patients [41]. Furthermore, PRUDENTE et al. [42] reported that BEC may have a long-term predictive value, as high BECs were associated with increased 9-year mortality in a Brazilian cohort of COPD patients.

Markers identifying exacerbated COPD patients at high risk are also clinically important outside of stable disease. According to the results of JøRGENSEN et al. [43], high serum soluble urokinase plasminogen activator receptor (suPAR) may highlight patients with an increased risk for 30-day mortality. Interestingly, PAPATHANASSIOU et al. [44] reported a significantly higher 1-year mortality in COPD patients who had low BECs at hospital admission. In contrast, low blood eosinophil levels were associated with lower rates of re-admission in a Russian study [45].

\section{The new GOLD ABCD severity grading system}

The updated GOLD 2017 classification of COPD severity grading generated debate and its impact was evaluated by several studies presented at the Congress. Compared to GOLD 2013, GOLD 2017 recommends a simplified $\mathrm{ABCD}$ assessment tool, using only exacerbation history and respiratory symptoms to guide treatment, while spirometry is used only for diagnosis and prognostic assessment [46]. Exclusion of spirometry from the $\mathrm{ABCD}$ assessment tool leads to the reclassification of a proportion of patients from GOLD groups $\mathrm{C}$ and $\mathrm{D}$ to groups $\mathrm{A}$ and $\mathrm{B}$. As a result, less intensive treatment is now recommended for these patients, who have significantly affected pulmonary function (FEV $1<50 \%$ 
predicted) but do not experience frequent exacerbations or burdensome symptoms at stable state. This approach is justified by the fact that currently available COPD pharmacotherapy targets respiratory symptoms and exacerbations but cannot delay lung function decline or prevent mortality. Therefore, it is anticipated that the newer GOLD classification will decrease the therapeutic burden of these patients without affecting their clinical outcomes. Application of GOLD 2017 criteria in a cross-sectional study involving over 19000 patients from the UK Clinical Practice Research Datalink (CPRD) led to reclassification of $37 \%$ of all participants from groups C and D (according to GOLD 2013) to groups A and B $[47,48]$. Only $16 \%$ of all patients with COPD were classified as GOLD groups C or D according to GOLD 2017, compared to 54\% according to GOLD 2013 [47, 48]. A cross-sectional study in Poland involving 2597 patients with COPD, as identified by primary care physicians, confirmed that the application of GOLD 2017 leads to a decrease in patients allocated to GOLD groups C and D by 60\% [49]. Less than $8 \%$ were classified as group C and $21 \%$ as group D. In the ECLIPSE cohort, only $7 \%$ and $15 \%$ of all participants were allocated to GOLD groups C and D, respectively, compared to $23 \%$ and $40 \%$ according to the GOLD 2013 classification [42]. Overall, application of GOLD 2017 criteria led to reclassification of $25-60 \%$ of all patients with COPD who were previously graded as GOLD groups $\mathrm{C}$ or $\mathrm{D}$, but were now classified as GOLD groups A or B.

The recently published 2019 version of the GOLD document suggests that the ABCD system should be used to guide the initial pharmacological management of COPD, while follow-up management should be guided by a review-assess-adjust cycle [50]. The previously described studies evaluated patients who were already receiving treatments that should have limited their symptoms and/or exacerbation burden. Therefore, they must have underestimated the participants' baseline ABCD grade (prior to treatment initiation) and this is a limitation. However, GOLD 2019 also suggests that treatment de-escalation may be needed in cases of ineffective treatment and side effects, but also of symptom resolution or overtreatment. The aforementioned studies reveal a significant proportion of patients who do not experience exacerbations and/or significant symptoms (GOLD groups A and B) and who are potentially overtreated, mostly due to their previous classification as GOLD groups $\mathrm{C}$ and D. For example, in the UK CPRD cohort, $22 \%$ and $43 \%$ of patients classified as GOLD stages A and B, respectively ( $26 \%$ of all patients with COPD), were receiving triple therapy. These patients represent a prime target for a treatment de-escalation trial aimed at limiting therapeutic burden and real-life data on the safety of de-escalation should be assessed.

Spirometry, which is not included in the GOLD 2017 ABCD grading, is an accurate predictor of mortality. For this reason, GOLD now suggests the use of the ABCD system to guide treatment, but the combination of both the ABCD system and spirometry for prognostic evaluation [46]. A number of studies assessed the predictive value of the ABCD system with regards to mortality. First, mortality was evaluated in the 784 participants of the real-life COPD cohort from the Czech Republic [51]. The shift of patients from GOLD groups C and D to groups A and B when using GOLD 2017 resulted in an increase in the mortality rate of group B (25\% compared to $18.7 \%$ with GOLD 2011) and group C (23.1\% compared to $17.9 \%$ with GOLD 2011). Secondly, in the ECLIPSE cohort, GOLD 2017 classification resulted in a trend towards increased mortality in all GOLD groups [40]. This suggests that the mortality rate of those reclassified was lower than that of patients who were consistently in groups $\mathrm{C}$ or D and higher than that of those who were consistently in groups A or B.

\section{Bronchiectasis}

Bronchiectasis is a highly complex condition characterised radiologically by the presence of pathological dilation of the airway and clinically by persistent cough, sputum production, recurrent respiratory tract infections and general malaise [52]. Bronchiectasis represents the final common pathway for a wide number of disease processes and is associated with numerous aetiologies and comorbidities, as well as a heterogeneous disease presentation, clinical course and response to treatment. The recently coined "vicious vortex" depicts the underlying pathophysiological interplay driving airway dysfunction, inflammation, infection and remodelling in bronchiectasis, with each pathophysiological process contributing to all the others [53]. Rather than breaking the "vicious cycle", which one would expect to halt disease progression, treatments such as antibiotics may target only one component of the vortex with the remaining components sustained by other stimuli, supporting the need for a multimodal therapeutic approach to this condition [53].

At this year's Congress, several large real-life cohort studies were presented using data from EMBARC, a prospective dataset of more than 11000 patients from more than 27 European countries. Marked heterogeneity in patient characteristics, management and outcomes were noted across different European countries, with significantly worse QoL, faster lung function decline and higher exacerbation frequency noted in Eastern Europe versus Western Europe, together with a significant between-country variability in 
access and referral to pulmonary rehabilitation [54, 55]. Classification of patients with nontuberculous mycobacteria (NTM) and primary ciliary dyskinesia (PCD), conditions deserving of disease-specific guidelines outside of bronchiectasis, showed older age, female sex, higher MRC dyspnoea scores and bilateral involvement of the middle lobe and lingua to be independently associated with the development of NTM in bronchiectasis, whilst PCD patients were typically younger at presentation with increased disease severity compared to age and sex-matched patients with idiopathic disease or bronchiectasis secondary to immune deficiency [56,57]. Bronchiectasis associated with inflammatory bowel disease (IBD), a recognised cause of bronchiectasis previously shown to be associated with a two-fold increase in mortality, showed no significant differences with regards to bronchiectasis severity or lung function decline and significantly lower rates of Pseudomonas colonisation in IBD patients compared to bronchiectasis due to other causes (perhaps suggesting that IBD may be skewed towards a more inflammatory rather than an infective pathophysiology) [58,59]. An analysis of determinants of QoL in bronchiectasis showed sputum production, breathlessness, exacerbations and chronic infection with Pseudomonas or NTM to be the greatest contributors to QoL burden [60]. Data from the Severe Asthma Network Italy (SANI) cohort showed that severe asthma patients with bronchiectasis had significantly reduced lung function, asthma control and QoL, with a higher exacerbation and hospitalisation frequency compared to those without bronchiectasis, suggesting a possible new phenotype [61].

Several high quality studies focusing on the science behind the disease were also presented, with multicentre studies looking at the effects of GORD and immune-allertypes in bronchiectasis as potential new disease endotypes. Having previously been shown to increase mortality 2.5 -fold, proteomics analysis of bronchiectasis patients with GORD demonstrated increased markers of epithelial injury and an increased defence response to bacterium, correlating with increased exacerbations and worse QoL compared to GORD-negative patients [59, 62]. Exploration of the role of allergy and sensitisation in bronchiectasis demonstrated a fungal driven immune-allertype to be associated with more severe disease and poorer lung function [63]. These are potentially treatable traits for a disease where limited treatment options currently exist.

BEDI et al. [64] showed that a pro-inflammatory mediator profile associated with dysregulation of LXA4 biosynthetic genes contributes to persistent infection in bronchiectasis. In addition, the Chalmers group showed that antimicrobial peptides and airway bacterial colonisation may be important in the pathogenesis of airway infection in bronchiectasis and that patients with severe bronchiectasis have reduced AMPK activation in sputum neutrophils, which may be associated with a significant reduction in ciliary beat frequency [65, 66]. Further work from this group showed a heterogeneous response of neutrophil phagocytosis to sputum supernatant, suggesting that airway factors inhibit neutrophil phagocytosis in some patients, resulting in worse brief symptom inventory (BSI) scores and poorer QoL [67]. Abnormal sputum rheology was observed in bronchiectasis airways compared to healthy airways but, perhaps surprisingly, no differences in sputum elasticity, viscosity or stiffness were noted in bronchiectasis patients with Pseudomonas aeruginosa (PA) versus those without $[68,69]$.

No approved therapies currently exist for bronchiectasis and many clinical trials have failed to meet their primary endpoints because of heterogeneous treatment responses and the inherent difficulty of identifying patients likely to respond. Post hoc analyses of ORBIT-3 and ORBIT-4 show that ARD-3150 (liposomal ciprofloxacin) is particularly effective in patients with four or more exacerbations in the previous year[70]. Similarly, serial Kaplan-Meier analyses showed the beneficial impact of ARD-3150 to be apparent from the third exacerbation in ORBIT-3 and for all serial exacerbations in ORBIT-4 [71]. Furthermore, the efficacy and safety profile was maintained during open-label extension [72].

While new therapies are welcomed, many are antibiotic-related and thus carry the risk of antibiotic resistance. Resistance acquisition was reported to occur with one in four bronchiectasis patients in a prospective observational multicentre study in Italy and Spain, with a correlating higher number of exacerbations and hospitalisations [73]. Whether this effect is maintained or intermittent requires further investigation.

Although disease phenotyping and endotyping are in their infancy in bronchiectasis they hold therapeutic promise for patients, leading towards our ultimate goal of administering more specialised and personalised medication.

\section{Small airways}

It is well established that the small airways are the major site of airway obstruction in COPD and in recent years have also been highlighted as a promising target for improving treatment outcomes in patients with poorly controlled asthma. The important role that small airways play in asthma was highlighted by Paul O'Byrne in a talk within an industry sponsored session. Professor O'Byrne indicated that airflow measurements such as $\mathrm{FEV}_{1}$ (which measure airflow in the larger airways) do not tend to correlate with 
disease severity, whereas uncontrolled asthma is associated with persistent small airways disease. Targeting the small airways through utilisation of extrafine particles, which have shown promising results in several studies, may be the key to improved asthma control.

The ERS also showcased the upcoming outcomes of the Assessment of Small Airways Involvement In Asthma (ATLANTIS) project. This project aims to better understand the role of small airways disease in asthma through determining the role of the small airways in clinical manifestations and to determine the best combination of diagnostic tests to apply [74]. Furthermore, the study has allowed further development and validation of a questionnaire specifically designed for assessing small airways disease [75]. Thanks to the collaborative work of a multinational consortium, the ATLANTIS study has shown for the first time how small airway abnormalities drive the clinical expression of asthma, both in a cross-sectional and a longitudinal manner, and results should help to optimise future patient care in asthma.

One key problem in investigating the role of the small airways in the pathophysiology of airway disease is effectively and accurately assessing their function. Ostridge et al. [76] presented a novel quantitative computed tomography (CT) method, disease probability measure (DPM), to assess the contribution of small airways disease in COPD. DPM measures pulmonary lobe density using inspiratory and expiratory images to measure the extent of air trapping. Patients were scanned and assessed as "normal", "emphysemous", or "gas trapping" due to small airway disease. Significantly more air trapping was found in COPD patients than in healthy subjects, ex-smokers and current smokers. This is a novel, precise way of measuring small airway contribution to disease pathology.

Potential causes of small airway disease development were also investigated and TANABE et al. [77] looked at the role that serine proteases may play in contributing to small airways disease. Lung tissues were collected from patients with COPD (GOLD stage 2) and asymptomatic control smokers and these were fixed and stained to measure protease activity. Interestingly, protease activity was significantly higher in COPD patients compared to control subjects and this may, therefore, be one of the factors behind airway remodelling. This finding prompted the authors to conclude that serine protease inhibitors might represent a potential therapeutic strategy against small airways disease in COPD.

As the small airways contribute significantly to the pathophysiology of asthma and COPD, many abstracts examined the optimal delivery method for therapies directly to them. BIDDISCOMBE et al. [78] compared the effect of tiotropium delivered from two different devices (a dry powder inhaler (DPI) and the slow mist inhaler (SMI) Respimat ${ }^{\mathrm{TM}}$ ) in 44 mild to moderate COPD patients. Physiological measures of both the small and large airways were taken to determine which device was better suited to delivering drugs to the lung periphery. Results showed that only the tiotropium SMI significantly improved small airway function and, furthermore, that it also positively impacted on large airway parameters, indicating that SMI formulation may be best at targeting both the large and small airways.

\section{Electronic health (e-Health)}

The digital evolution in respiratory medicine as an innovative and personalised approach [79] was depicted by Omar Usmani in a highly attended symposium. In particular, the speaker presented the study protocol $[80,81]$ and the preliminary findings of a multicentre, Horizon 2020 European Union funded project started on January 2015 which is close to completion (www.myaircoach.eu/myaircoach/).

One of the main goals of the project is to help patients to manage their asthma through a personalised e-Health approach, using user-friendly tools to increase patients' awareness of their clinical state, as well as adherence to medical treatments and the effectiveness thereof. MyAirCoach sensors have been monitoring and storing several physiological, behavioural and environmental parameters which will be analysed and crossed with asthma data. Thanks to sophisticated computational modelling techniques, MyAirCoach will be able to provide measurements, features, indicators and personal profile data to give a picture of a patient's condition and ensure clinical state awareness and optimal treatment. In the near future, healthcare professionals could use MyAirCoach to supervise the condition of their patients and adjust their prescribed medication accordingly. MyAirCoach will provide clinicians with early indications of increasing asthma symptoms or exacerbations, while helping to understand the mechanisms underpinning the progression of the disease. The impact of MyAirCoach is expected to set the basis for the widespread adoption of e-Health self-management systems across the spectrum of respiratory diseases.

\section{Airway sensory nerves and cough}

Cough is a reflex event initiated following activation of airway sensory nerves and is a major symptom of airway diseases such as asthma and COPD; however, it can also be idiopathic in origin [82]. When cough lasts for more than 8 weeks it is known as chronic cough, which is a major unmet medical need that lowers the QoL of sufferers [83]. The cough reflex was again a topic of great interest at Congress, 
highlighted by the number of extremely well attended abstract sessions dedicated to cough or neural control of the airways and also a symposium entitled "Towards understanding and managing chronic cough", which contained lectures from many well-known names in the cough field.

Airway sensory nerves housed within the vagus nerve initiate airway reflexes such as cough [84] and a number of studies highlighted the potential role of neuronal remodelling in the pathogenesis of cough and airway hyperresponsiveness (AHR) in airway disease. An abstract by BLUM et al. [85] utilised bronchoscopic biopsies from subjects with healthy control and immunolabelled sections for nerves and eosinophils. The authors showed that the samples from chronic cough patients had a doubling of nerve length as well as increased numbers of branch points compared to healthy controls and suggest that increased sensory nerve density may underlie the development of cough hypersensitivity. A further study presented by KistemaKer et al. [86] utilised ovalbumin (OVA) sensitised mice that were challenged either acutely or chronically. AHR was then measured in control and vagotomised subjects, and contraction to methacholine $(\mathrm{MCh})$ and electrical field stimulation (EFS) were measured in lung slices. The authors showed that following chronic OVA challenge, AHR in vagotomised animals was attenuated and protein gene product 9.5 (PGP 9.5) expression was increased; however, there were no changes following acute challenge. Furthermore, EFS-induced contraction was increased but MCh-induced contraction remained unchanged with an increase of brain-derived neurotrophic factor (BDNF) expression in lung tissue. These studies indicate that airway sensory nerves may contribute to the pathogenesis of airway diseases, along with the increased cough hypersensitivity seen and they highlight a novel target for treatment.

Receptors on airway sensory nerves are key targets for the treatment of neuronally driven symptoms such as cough. CHEN et al. [87] presented an abstract looking at the activation of an oxidant sensor ion channel, transient receptor potential ankyrin 1 (TRPA1), on sensory nerves. Using vagus nerves from humans, guinea pigs and TRPA1-knockout mice it was shown that there are a number of activators of TRPA1, both endogenous (e.g. the disease-relevant mediator prostaglandin $\mathrm{E}_{2}\left(\mathrm{PGE}_{2}\right)$ ) and exogenous (e.g. acrolein), which can work to activate the channel via distinct mechanisms. CHEN et al. showed that $\mathrm{PGE}_{2}$ activated TRPA1 through the activation of NADPH oxidase, but acrolein is a more direct cause of reactive oxygen species (ROS) as activation was inhibited by N-acetylcysteine (NAC). This highlights different modalities of TRPA1 activation which may provide targets for treatment. In addition, WoRTLEY et al. [88] were able to show that a key asthma treatment, $\beta$-agonists, can inhibit airway sensory nerve activation and cough in a guinea pig model. Furthermore, this effect is not lost with chronic treatment, unlike the effect on bronchoconstriction, highlighting that $\beta$-agonists may still be a useful long-term treatment for cough even when efficacy is reduced against bronchoconstriction.

The P2X3 inhibitor MK7624 has been shown to be the most promising novel cough therapeutic in recent years, with a preliminary study [89] indicating that it was able to cause a $75 \%$ reduction in daytime cough frequency, albeit with a side effect of taste disturbance. SMITH et al. [90] presented an update on MK7624, investigating efficacy responses and evaluating baseline and demographic characteristics. The primary end point of this study was awake cough frequency (ACF) and, from 253 patients, the authors showed that ACF was significantly reduced by MK7624 and also that ACF was lower in the USA compared to the UK. BIRRING et al. [91] presented a further abstract on cough demographics that looked at patient characteristics and triggers eliciting refractory chronic cough. The research indicated that those suffering from treatment refractory chronic cough were mostly female of middle/older age. Furthermore, although cough triggers were unpredictable, a high proportion of triggers were associated with neuronal hypersensitivity. These studies highlight that patients demographics are an important factor when evaluating the efficacy of novel cough therapeutics.

Along with the number of high quality abstracts addressing the problem of chronic cough, cough has further been highlighted by the creation in 2018 of a novel ERS Taskforce. This is a clinical research collaboration entitled "Neurocough: new understanding in the treatment of chronic cough", a pan-European multicentre network that aims to improve the management of cough.

Conflict of interest: F. Schleich has nothing to disclose. A. Bikov has nothing to disclose. A.G. Mathioudakis reports grants and personal fees from Boehringer Ingelheim, and personal fees from GlaxoSmithKline, outside the submitted work. M. McDonnell reports grants from the Health Research Board, Ireland, outside the submitted work. C. Andersson has nothing to disclose. M. Bonini has nothing to disclose. L. Uller has nothing to disclose. M. Idzko has nothing to disclose. D. Singh reports grants and personal fees from AstraZeneca, Boehringer Ingelheim, Chiesi, GlaxoSmithKline, Glenmark, Menarini, Mundipharma, Novartis, Pfizer, Pulmatrix, Therevance and Verona, and personal fees from Cipla and Peptinnovate, outside the submitted work. J.L. Lopez-Campos reports personal fees and nonfinancial support from Boehringer, Grifols, Novartis, Chiesi, AstraZeneca and GSK, personal fees from Teva, Rovi, Ferrer, Bial, Esteve and Gebro Pharma, and grants, personal fees and nonfinancial support from Menarini, outside the submitted work. A. Bossios reports personal fees and other support for consultancy from AstraZeneca, Teva and GSK, outside the submitted work. I.M. Adcock has nothing to disclose. O. Usmani reports grants and personal fees from AstraZeneca, Boehringer Ingelheim and Chiesi, personal fees from Aerocrine, Napp, Mundipharma, Sandoz, Prosonix, Takeda, 
Zentiva Cipla and Pearl Therapeutics, and grants from GlaxoSmithKline and Edmond Pharma, outside the submitted work. A. Spanevello has nothing to disclose. S.J. Bonvini has nothing to disclose.

\section{References}

1 World Health Organization. Chronic respiratory diseases: asthma. www.who.int/respiratory/asthma/en/.

2 Pavord ID, Bush A, Holgate S. Asthma diagnosis: addressing the challenges. Lancet Respir Med 2015; 3: 339-341.

3 Wenzel SE. Asthma: defining of the persistent adult phenotypes. Lancet 2006; 368: 804-813.

4 Nawijn M, Carpaij O, Viera Braga F, et al. Novel cell types and altered cell states in asthma revealed by single-cell RNA sequencing of airway wall biopsies. Eur Respir J 2018; 52: Suppl. 62, OA505.

5 Tokunaga Y, Imaoka H, Morimatsu Y, et al. Role of CD63 in human fatal asthma and murine asthma model. Eur Respir J 2018; 52: Suppl. 62, PA648.

6 Silberbrandt A, Vin Bulow A, Backer V, et al. Airway eosinophilia in severe asthma is associated with smoking history. Eur Respir J 2018; 52: Suppl. 62, PA948.

7 Mistry H, Freeman A, Thirwall Y, et al. Does the nature of adult difficult asthma differ by age of onset? Findings from WATCH. Eur Respir J 2018; 52: Suppl. 62, PA1086.

8 Mathieu E, Escribano-Vazquez U, Descamps D, et al. Paradigms of lung microbiota functions in health and disease, particularly, in asthma. Front Physiol 2018; 9: 1168.

9 Potaczek DP, Harb H, Alhamwe BA, et al. Interleukin-6 is important for the development of Acinetobacter lwoffii-mediated protection against asthma. Eur Respir J 2018; 52: Suppl. 62, PA1108.

10 Anatriello E, Britto A, Albertina R, et al. Lactobacillus bulgaricus reduces the expression of Toll-like 4 receptor on dendritic cells and attenuates the airway inflammation in asthmatic mice. Eur Respir J 2018; 52: Suppl. 62, PA1107.

11 De Boer GM, Braunstahl GJ, Hendriks RW, et al. Bacterial lysates in the prevention of asthma exacerbations in uncontrolled asthma: the Breathe study. Eur Respir J 2018; 52: Suppl. 62, PA5008.

12 Chapman KR, Boulet LP, Rea RM, et al. Suboptimal asthma control: prevalence, detection and consequences in general practice. Eur Respir J 2008; 31: 320-325.

13 van der Meer A, De Jong K, Bel EH, et al. Dynamic hyperinflation predicts impaired daily life activity in asthma. Eur Respir J 2018; 52: Suppl. 62, PA4001.

14 Backman H, Hedman L, Stridsman C, et al. Eosinophilic inflammation and lung function decline in a long-term follow-up of a large population-based asthma cohort. Eur Respir J 2018; 52: Suppl. 62, OA294.

15 Graff S, Moermans C, Louis R, et al. Impact of eosinophilia and time on lung function decline in adult asthma. Eur Respir J 2018; 52: Suppl. 62, PA2017.

16 Gamble J, Stevenson M, McClean E, et al. The prevalence of nonadherence in difficult asthma. Am J Respir Crit Care Med 2009; 180: 817-822.

17 Alahmadi F, Simpson A, Gomez C, et al. Measures of adherence in patients with severe asthma prescribed systemic steroids in the U-BIOPRED cohort. Eur Respir I 2018; 52: Suppl. 62, PA3992.

18 Azim A, Mistry H, Harvey MA, et al. Comorbidities in the difficult asthma clinic: results from WATCH. Eur Respir J 2018; 52: Suppl. 62, PA1087.

19 McDonald VM, Hiles SA, Godbout K, et al. Treatable traits can be identified in a severe asthma registry and predict future exacerbations. Respirology 2019; 24: 37-47.

20 Chen E, Miller GE. Stress and inflammation in exacerbations of asthma. Brain Behav Immun 2007; 21: 993-999.

21 Bacon S, Plourde A, Paine N, et al. Impact of acute active and passive stress on physiological responses in adults with asthma. Eur Respir J 2018; 52: Suppl. 62, PA654.

22 Green RH, Brightling CE, Woltmann G, et al. Analysis of induced sputum in adults with asthma: identification of subgroup with isolated sputum neutrophilia and poor response to inhaled corticosteroids. Thorax 2002; 57: 875-879.

23 Marcon A, Marchetti P, Anto JM, et al. Inhaled corticosteroids and FEV1 decline in asthma: an international cohort study. Eur Respir J 2018; 52: Suppl. 62, OA295.

24 de Marco R, Marcon A, Jarvis D, et al. Inhaled steroids are associated with reduced lung function decline in subjects with asthma with elevated total IgE. J Allergy Clin Immunol 2007; 119: 611-617.

25 Hanania N, Fang J, Wei Z, et al. Real-world effectiveness of omalizumab in allergic asthma patients with low or high blood eosinophils: a retrospective claims analysis study. Eur Respir J 2018; 52: Suppl. 62, PA1029.

26 Ortega H, Meyer E, Brusselle G, et al. Immunogenicity of mepolizumab in patients with severe eosinophilic asthma: experience from the clinical development program. Eur Respir J 2018; 52: Suppl. 62, OA1650.

27 Wenzel S, Brusselle G, Hirsch I, et al. Benralizumab efficacy in patients with uncontrolled eosinophilic asthma by age at diagnosis. Eur Respir J 2018; 52: Suppl. 62, PA603.

28 Corren J, Castro M, Maspero JF. Dupilumab improves asthma-related patient reported outcomes in asthma patients with chronic rhinosinusitis or nasal polyposis (CRS/NP) in LIBERTY ASTHMA QUEST. Eur Respir J 2018; 52: Suppl. 62, PA1124.

29 Torrego A, Herth F, Munoz AM, et al. Bronchial thermoplasty global registry: one year results. Eur Respir J 2018; 52: Suppl. 62, OA1921.

30 Frix A, Heinen V, Schleich F, et al. Bronchial thermoplasty in severe asthmatics: a retrospective study on 10 cases with one year follow-up. Eur Respir J 2018; 52: Suppl. 62, PA605.

31 Goorsenberg AWM, D'Hooghe JNS, Slats AM, et al. The effect of bronchial thermoplasty on lung function in severe asthma patients. Eur Respir J 2018; 52: Suppl. 62, OA3560.

32 Sano A, Sano H, Iwanaga $\mathrm{T}$, et al. The usefulness of the impulse oscillometry for predicting the treatment outcomes of bronchial thermoplasty in patients with severe asthma. Eur Respir J 2018; 52: Suppl. 62, PA3694.

33 Bafadhel M, Pavord ID, Russell REK. Eosinophils in COPD: just another biomarker? Lancet Respir Med 2017, 5: 747-759.

34 Pascoe S, Barnes N, Brusselle MT, et al. Late breaking abstract - blood eosinophil counts and treatment response in COPD: analyses of IMPACT. Eur Respir J 2018; 52: Suppl. 62, OA2127.

35 Burge PS, Calverley PM, Jones PW, et al. Randomised, double blind, placebo controlled study of fluticasone propionate in patients with moderate to severe chronic obstructive pulmonary disease: the ISOLDE trial. BMJ 2000; 320: 1297-1303 
Mathioudakis A, Foden P, Vestbo J. Late breaking abstract - blood eosinophil count (EOS) can accurately predict responsiveness to inhaled corticosteroids (ICS) in COPD, but only if measured while patients are not receiving steroids. Eur Respir J 2018; 52: Suppl. 62, OA2125.

37 Hurst J, Kostikos K, Chapman KR, et al. Persistent blood eosinophilia and COPD exacerbation risk after ICS withdrawal from triple therapy in the SUNSET study. Eur Respir J 2018; 52: Suppl. 62, OA5387.

38 Karch A, Vogelmeier C, Welte T, et al. The German COPD cohort COSYCONET: aims, methods and descriptive analysis of the study population at baseline. Respir Med 2016; 114: 27-37.

39 Waschki B, Alter P, Zeller T, et al. Late breaking abstract - high-sensitivity troponin I predicts all-cause mortality in stable COPD in the COSYCONET cohort. Eur Respir J 2018; 52: Suppl. 62, OA2138.

40 Vestbo J, Anderson W, Coxson HO, et al. Evaluation of COPD longitudinally to identify predictive surrogate end-points (ECLIPSE). Eur Respir J 2008; 31: 869-873.

41 Canet MRF, Noell G, Bakke P, et al. Longitudinal stability and association with all-cause mortality of the 2017 GOLD groups in the ECLIPSE cohort. Eur Resp J 2018; 52: Suppl. 62, OA2140

42 Prudente R, Mesquita C, Ferrari R, et al. Peripheral eosinophils and mortality in COPD patients over nine years Eur Respir J 2018; 52: Suppl. 62, OA5383.

43 Jørgensen DV, Godtfredsen NS, Marsaa K, et al. Soluble urokinase plasminogen activator receptor (suPAR) predicts mortality in exacerbated COPD. Eur Respir J 2018; 52: Suppl. 62, OA2142.

44 Papathanassiou E, Papaioannou A, Hillas G, et al. Eosinophils and acute exacerbation of COPD: a potent biomarker for patients at increased risk. Preliminary reports. Eur Respir J 2018; 52: Suppl. 62, PA2016.

45 Soe AK, Kevorkova MS, Nuralieva GS, et al. Blood eosinophilia (BE) and outcomes in patients with acute sever exacerbations of COPD (AECOPD). Eur Respir J 2018; 52: Suppl. 62, OA5382.

46 Vogelmeier CF, Criner GJ, Martinez FJ, et al. Global strategy for the diagnosis, management, and prevention of chronic obstructive lung disease 2017 Report: GOLD executive summary. Eur Respir J 2017; 49 : 1700214.

47 Gayle A, Dickinson S, Morris $\mathrm{K}$, et al. What is the impact of GOLD 2017 recommendations in primary care? A descriptive study of patient classifications, treatment burden and costs. Int J Chron Obstruct Pulmon Dis 2018 13: 3485-3492.

48 Mathioudakis AG, Vestbo J, Gayle A, et al. GOLD ABCD assessment tool: comparison of 2013 and 2017 classifications in the UK Clinical Practice Research Datalink (CPRD). Eur Respir J 2018; 52: Suppl. 62, PA2011.

49 Brozek G, Nowak N, Mateusz J, et al. The same COPD patients, different GOLD criteria - what are the consequences of changing GOLD 2007-2011-2017 recommendations? Eur Respir J 2018; 52: Suppl. 62, PA1164.

50 Global strategy for the diagnosis, management and prevention of chronic obstructive pulmonary disease (2019 report). Global Initiative for Chronic Obstructive Pulmonary Disease (GOLD), 2019. https://goldcopd.org/wpcontent/uploads/2018/11/GOLD-2019-v1.7-FINAL-14Nov2018-WMS.pdf

51 Plutinsky M, Brat K, Koblizek V, et al. Implementation of the GOLD 2017 disease classification in a real-life COPD cohort. Eur Respir J 2018; 52: Suppl. 62, PA3858.

52 Chalmers JD, Aliberti S, Blasi F. Management of bronchiectasis in adults. Eur Respir J 2015; 45: 1446-1462.

53 Flume PA, Chalmers JD, Olivier KN. Advances in bronchiectasis: endotyping, genetics, microbiome, and disease heterogeneity. Lancet 2018; 392: 880-890.

54 Chalmers JD, Polverino E, Blasi F, et al. The heterogeneity of bronchiectasis patient characteristics, management and outcomes across Europe: data from the EMBARC registry. Eur Respir J 2018; 52: Suppl. 62, PA2676.

55 Walker P, Herrero B, Spinou A, et al. Variability in access and referral to pulmonary rehabilitation in European bronchiectasis patients enrolled in the EMBARC registry. Eur Respir J 2018; 52: Suppl. 62, OA5201.

56 Loebinger M, Ringshausen F, Haworth C, et al. Characteristics of patients with pulmonary non-tuberculous mycobacterial infection in bronchiectasis: data from the EMBARC registry. Eur Respir J 2018; 52: Suppl. 62, PA348.

57 Shoemark A, Polverino E, Blasi F, et al. Primary ciliary dyskinesia in adults with bronchiectasis: data from the Embarc registry. Eur Respir J 2018; 52: Suppl. 62, PA359.

58 De Soyza A, Chalmers JD, Dimakou K, et al. Impact of inflammatory bowel disease in bronchiectasis (IBD-BR) data from the EMBARC registry. Eur Respir J 2018; 52: Suppl. 62, PA2678.

59 McDonnell MJ, Aliberti S, Goeminne PC, et al. Comorbidities and the risk of mortality in patients with bronchiectasis: an international cohort study. Lancet Respir Med 2016; 4: 969-979.

60 Polverino E, Blasi F, Ringshausen F, et al. Determinants of quality of life in bronchiectasis using the quality of life bronchiectasis (QOL-B) questionnaire: data from the EMBARC registry. Eur Respir J 2018; 52: Suppl. 62, OA4951.

61 Heffler EM, Aliberti S, Sirena C, et al. Impact of bronchiectasis on severe asthma: data from the Severe Asthma Network Italy (SANI) registry. Eur Respir J 2018; 52: Suppl. 62, PA5494.

62 McDonnel MJ, Das J, O’Toole D, et al. Effects of gastro-oesophageal reflux and pulmonary micro-aspiration in bronchiectasis. Eur Respir J 2018; 52: Suppl. 62, OA4950.

63 Tiew PY, Aogain MM, Lim AYH, et al. Immuno-allertypes in non-cystic fibrosis bronchiectasis. Eur Respir J 2018 52: Suppl. 62, PA1142.

64 Bedi P, Davidson D, McHigh B, et al. Dysregulation of lipid mediators in clinically stable bronchiectasis. Eur Respir J 2018; 52: Suppl. 62, PA2646.

65 Sibila Vidal O, Perea L, Cantó E, et al. Antimicrobial peptides and airway bacterial colonization in bronchiectasis Eur Respir J 2018; 52: Suppl. 62, OA4948.

66 Giam YH, Shoemark A, Keir H, et al. AMPK is inhibited in severe bronchiectasis and may relate to reduced ciliary beat frequency. Eur Respir J 2018; 52: Suppl. 62, PA351.

67 Keir RH, Cassidy D, Crichton M, et al. Heterogeneous impact of sputum supernatant on neutrophil phagocytosis in bronchiectasis. Eur Respir J 2018; 52: Suppl. 62, OA4949.

68 Ramsey K, Radicioni G, Hill D, et al. Airways mucus pathogenesis in patients with non-cystic fibrosis bronchiectasis. Eur Respir J 2018; 52: Suppl. 62, PA5049.

69 Alcaraz Serrano V, Llorens-Llacuna J, Gimeno-Santos E, et al. Does Pseudomonas aeruginosa infection alter sputum viscoelastic properties in bronchiectasis patients? Eur Respir J 2018; 52: Suppl. 62, PA1461.

70 Chalmers JD, Thompson B, Froehlich J, et al. Effect of prior year pulmonary exacerbation frequency on response to ARD-3150 in patients with non-cystic fibrosis bronchiectasis chronically infected with Pseudomonas aeruginosa. Eur Respir J 2018; 52: Suppl. 62, PA2675. 

analyses. Eur Respir J 2018; 52: Suppl. 62, OA4954. O'Donnell A, Gonda I, Cipolla D, et al. Open-label extension (OLE) of ORBIT-3 and ORBIT-4 trials of ARD-3150 in non-cystic fibrosis bronchiectasis (NCFB). Eur Respir J 2018; 52: Suppl. 62, PA357.

73 Faverio P, Bonaiti G, Zucchetti S, et al. Development of antibiotic-resistance in P. aeruginosa obtained from bronchiectasis patients: a multicentre prospective observational study. Eur Respir J 2018; 52: Suppl. 62, PA5314.

74 Postma DS, Brightling C, Fabbri L, et al. Unmet needs for the assessment of small airways dysfunction in asthma: introduction to the ATLANTIS study. Eur Respir J 2015; 45: 1534-1583.

75 Schiphof-Godart L, van der Wiel E, Ten Hacken N, et al. Development of a tool to recognize small airways dysfunction in asthma (SADT). Health Qual Life Outcomes 2014; 12: 155.

76 Ostridge K, Gove K, Kirby M, et al. Using DPM CT analysis to assess the contribution of small airways disease in COPD. Eur Respir J 2018; 52: Suppl. 62, OA3795.

77 Tanabe N, Sato A, Mizutani T, et al. Protease anti-protease imbalance and small airways disease in COPD. Eur Respir J 2018; 52: Suppl. 62, PA4256.

78 Biddiscombe M, Saleem A, Meah S, et al. Efficacy of the device in targeting tiotropium to the small airways in COPD. Eur Respir J 2018; 52: Suppl. 62, PA1017.

79 Bonini M, Usmani OS. Novel methods for device and adherence monitoring in asthma. Curr Opin Pulm Med 2018; 24: 63-69.

80 Honkoop PJ, Simpson A, Bonini M, et al. MyAirCoach: the use of home-monitoring and mHealth systems to predict deterioration in asthma control and the occurrence of asthma exacerbations: study protocol of an observational study. BMJ Open 2017; 7: e013935.

81 Simpson AJ, Honkoop PJ, Kennington E, et al. Perspectives of patients and healthcare professionals on mHealth for asthma self-management. Eur Respir J 2017; 49: 1601966.

82 Morice AH, Fontana GA, Belvisi MG, et al. ERS guidelines on the assessment of cough. Eur Respir J 2007; 29: $1256-1276$.

83 French CL, Irwin RS, Curley FJ, et al. Impact of chronic cough on quality of life. Arch Intern Med 1998; 158: $1657-1661$

84 Canning BJ, Mori N, Mazzone SB. Vagal afferent nerves regulating the cough reflex. Respir Physiol Neurobiol 2006; 152: 223-242.

85 Blum E, Drake M, Fryer A, et al. Airway sensory innervation is increased in chronic cough. Eur Respir J 2018 52: Suppl. 62, OA3565.

86 Nijboer S, De Groot AP, VohiDalova E, et al. Allergen-induced BDNF expression and neuronal remodelling contributes to airway hyperresponsiveness. Eur Respir J 2018; 52: Suppl. 62, PA5262.

87 Chen X, Bonvini SJ, Dubuis E, et al. Characterisation of TRPA1 activation on sensory nerves. Eur Respir J 2018, 52: Suppl. 62, PA5263.

88 Wortley MA, Bonvini SJ, Flajolet PLM, et al. The anti-tussive effects of an inhaled LABA are maintained after chronic treatment. Eur Respir J 2018; 52: Suppl. 62, PA5261.

89 Abdulqawi R, Dockry R, Holt K, et al. P2X3 receptor antagonist (AF-219) in refractory chronic cough: a randomised, double-blind, placebo controlled phase 2 study. Lancet 2015; 286: 244-245.

90 Smith JA, McGarvey LP, Morice AH, et al. The effect of baseline factors on treatment response with MK-7624, a P2X3 antagonist, in refractory chronic cough. Eur Respir J 2018; 52: Suppl. 62, PA811.

91 Birring SS, Muccino DR, Morice AH, et al. Patient characteristics and triggers eliciting chronic cough. Eur Respir J 2018; 52: Suppl. 62, PA820. 